\title{
The Impact of Meta-analyses on Medical Decisions
}

\author{
Jose G. Franco Jr ${ }^{1}$, João Batista A. Oliveira²
}

${ }^{1}$ President of SBRA - Brazilian Society of Assisted Reproduction/Scientific Director of Center for Human Reproduction Prof. Franco Jr, Ribeirao Preto, SP, Brazil

${ }^{2}$ Center for Human Reproduction Prof. Franco Jr, Ribeirao Preto, SP, Brazil

Evidence-based medicine has elevated meta-analyses to the status of key parameter in medical decision-making (Sigman, 2011; Humaidan \& Polyzos, 2012; Walker et al., 2008). Every year, over 6,000 meta-analyses are published in medical journals and submitted to the appreciation of physicians, who then have to analyze their conclusions and use them to support medical decisions. ART does not escape this rule, and meta-analyses concerning topics ripe with controversy - PGS (Preimplantation Genetic Screening), GnRH agonists vs. GnRH antagonists, endometrial injury, androgen therapy for women with poor ovarian response, etc. - are frequently published.

On the other hand, some meta-analyzes have conclusions that periodically change with the addition of only one new prospective randomized trial; we could say it is on the borderline of statistical significance. This also adds to the uncertainty physicians are faced with because the idea of accepting or not the use of the methodology in question is periodically changing.

The following general principles may be useful:

1. Base your medical decisions on meta-analyses including at least five prospective randomized trials.

2. Refer to meta-analyses including at least 1,000 individuals, 500 in the case and 500 in the control group.

3. From the standpoint of statistics, in addition to the significance of the differences between case and control groups, heterogeneity cannot be significant and inconsistency (I2) should be kept at $50 \%$ or under. Excessive heterogeneity indicates issues such as divergent enrollment criteria, inconsistent definitions for the studied variables, etc.

4. Figure 1 shows the description of a meta-analysis (Dentali et al., 2007) considered ideal to support medical decisions on the use of anticoagulants to prevent pulmonary embolism (statistically significant difference between case and control groups: chi-square $=10.95 ; \quad P=0.0009$; non-significant Cochran's Q (heterogeneity); and I2 =0).

Figure 1. Anticoagulation prevents pulmonar embolism original meta-analysis (Dentali et al., 2007)

Relative risk meta-analysis plot (fixed effects)

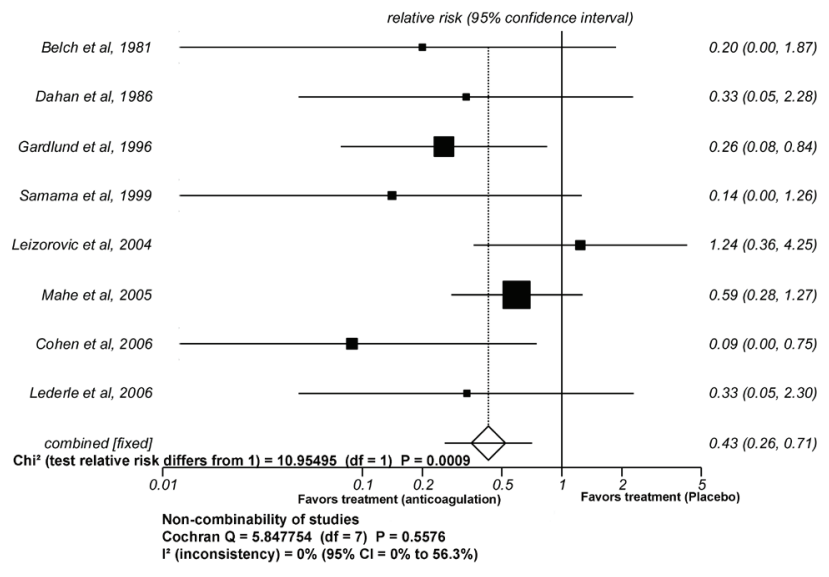

5. The credibility test may be used to assess the level of instability of the conclusions reported in the meta-analysis:

Present: the randomized removal of one or two papers does not affect the conclusion of the meta-analysis.

Absent: the randomized removal of one paper affects the conclusion of the meta-analysis.

Figure 2 shows conclusions of meta-analyses that remained unchanged after the removal of one (2A: Mahe et al., 2005) and two papers (2B: Belch et al., 1981; Dahan et al., 1986); only inconsistency moved to a still low $7.7 \%$.

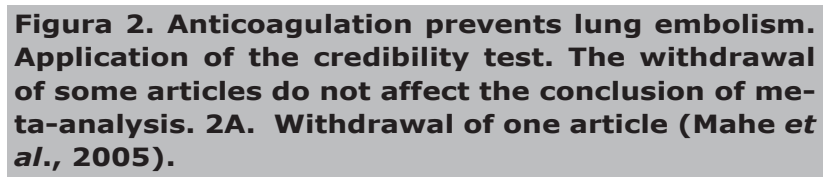
al., 2005).

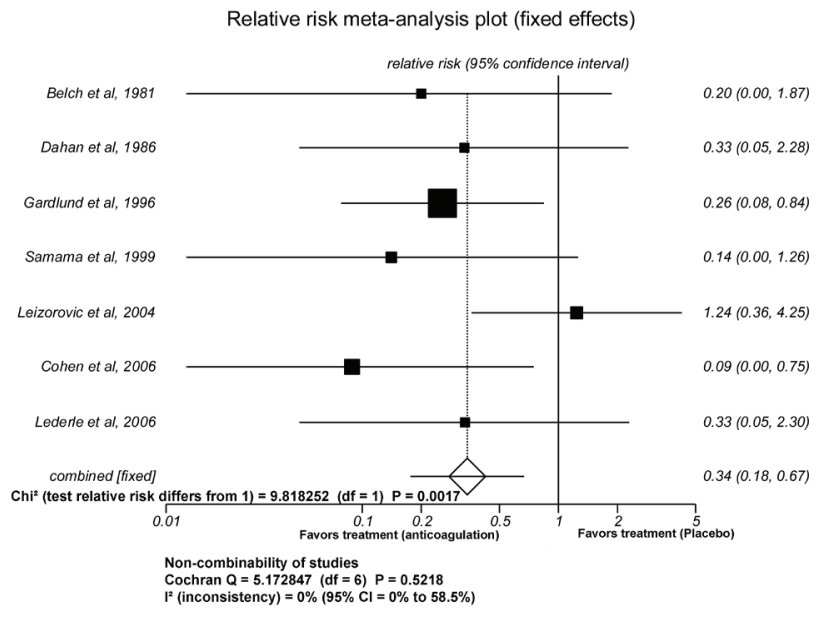

2B. Withdrawal de two articles (Belch et al., 1981; Dahan et al., 1986).

Relative risk meta-analysis plot (fixed effects)

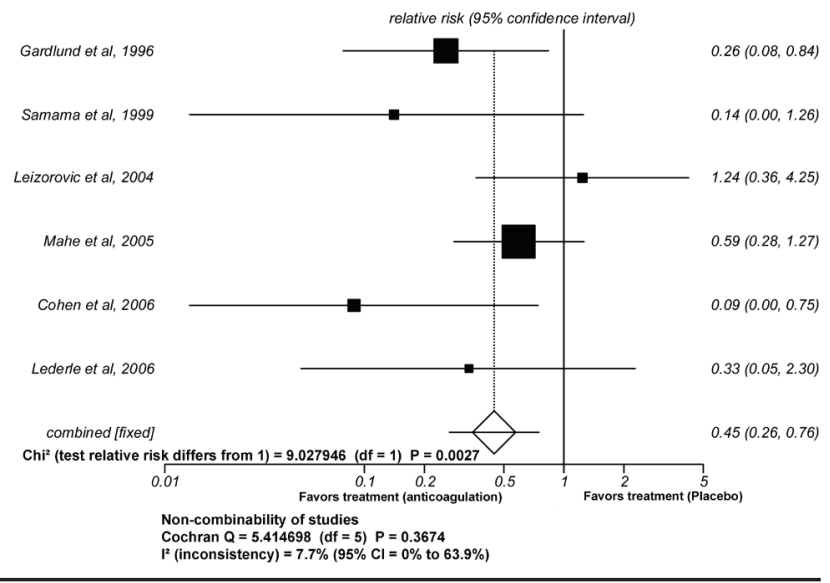


Finally, remember to study each of the papers included in the meta-analysis. They are the backbone supporting the conclusions used as references in medical decision-making. It is better to base your decisions on an excellent prospective randomized trial than on a meta-analysis reporting dubious conclusions.

\section{REFERENCES}

Belch JJ, Lowe GD, Ward AG, Forbes CD, Prentice CR. Prevention of deep vein thrombosis in medical patients by low-dose heparin. Scott Med J. 1981;26:115-7.

Cohen AT, Davidson BL, Gallus AS, Lassen MR, Prins MH, Tomkowski W, Turpie AG, Egberts JF, Lensing AW; ARTEMIS Investigators. Efficacy and safety of fondaparinux for the prevention of venous thromboembolism in older acute medical patients: randomised placebo controlled trial. BMJ. 2006;332:325-9.

Dahan R, Houlbert D, Caulin C, Cuzin E, Viltart C, Woler M, Segrestaa JM. Prevention of deep vein thrombosis in elderly medical in-patients by a low molecular weight heparin: a randomized double-blind trial. Haemostasis. 1986;16:159-64.

Dentali F, Douketis JD, Gianni M, Lim W, Crowther MA. Meta-analysis: anticoagulant prophylaxis to prevent symptomatic venous thromboembolism in hospitalized medical patients. Ann Intern Med. 2007;146:278-88.

Gardlund B. Randomised, controlled trial of low-dose heparin for prevention of fatal pulmonary embolism in patients with infectious diseases. The Heparin Prophylaxis Study Group. Lancet. 1996; 347:1357-61.
Humaidan P, Polyzos NP. (Meta)analyze this: Systematic reviews might lose credibility. Nat Med. 2012;18:1321.

Leizorovicz A, Cohen AT, Turpie AG, Olsson CG, Vaitkus PT, Goldhaber SZ. Randomized, placebo-controlled trial of dalteparin for the prevention of venous thromboembolism in acutely ill medical patients. Circulation. 2004;110: 8749.

Lederle FA, Sacks JM, Fiore L, Landefeld CS, Steinberg N, Peters RW, Eid AA, Sebastian J, Stasek JE Jr, Fye CL. The prophylaxis of medical patients for thromboembolism pilot study. Am J Med. 2006;119:54-9.

Mahe I, Bergmann JF, d'Azemar P, Vaissie JJ, Caulin C. Lack of effect of a low-molecular-weight heparin (nadroparin) on mortality in bedridden medical in-patients: a prospective randomised double-blind study. Eur J Clin Pharmacol. 2005;61:347-51.

Samama MM, Cohen AT, Darmon JY, Desjardins L, Eldor A, Janbon C, Leizorovicz A, Nguyen H, Olsson CG, Turpie AG, Weisslinger N. A comparison of enoxaparin with placebo for the prevention of venous thromboembolism in acutely ill medical patients. Prophylaxis in Medical Patients with Enoxaparin Study Group. N Engl J Med. 1999;341:793800.

Sigman M, A meta-analysis of meta-analyses Fertil Steril. 2011;96:11-4.

Walker E, Hernandez AV, Kattan MW. Meta-analysis: Its strengths and limitations. Cleve Clin J Med. 2008;75:431-9. 